\title{
Involvement of Palliative Care Services Strongly Predicts Place of Death in Belgium
}

\author{
Dirk Houttekier, M.A., Joachim Cohen, Ph.D.,' Lieve Van den Block, Ph.D., \\ Nathalie Bossuyt, M.D., ${ }^{2}$ and Luc Deliens, Ph.D., ${ }^{1,3}$
}

\begin{abstract}
Introduction: Place of death is considered a quality indicator of end-of-life care and enabling people to die were they choose is an important aspiration of palliative care. This study aims to examine the association between involvement of palliative care services and place of death.

Methods: Data about patient characteristics, use of general health care, and involvement of palliative care services in nonsudden or expected deaths in all health care settings in 2005-2006 $(N=1690)$ were collected by a surveillance network of general practitioners (GPs) in Belgium. Bivariate and multivariate associations between involvement of palliative care services and dying at home, in hospital, in a care home, or in a palliative care unit were examined using $\chi^{2}$ tests and Wald tests.

Results: Palliative care services were involved in $21.8 \%$ of deaths of those living at home, in $29.1 \%$ of those living in care homes, and in $12.4 \%$ of deaths in hospital. People were more likely to die in their usual residence rather than in hospital if multidisciplinary palliative home care teams (odds ratio [OR]: 8.4, confidence interval [CI]: 4.7-15.1) or the palliative care reference persons of their care home (OR: 9.4, CI: 3.3-26.7) were involved. Involvement of multidisciplinary palliative support teams in hospitals was associated with lower chances of dying at home (OR: 0.3, CI: 0.1-0.9). High involvement of GPs was not directly associated with out-of-hospital death.

Discussion: Involving multidisciplinary palliative home care teams and palliative care reference persons in care homes could support people in dying out-of-hospital. Health care policy-makers should consider strategies to improve involvement of palliative care services in all health care settings.
\end{abstract}

\section{Introduction}

E NABLING PEOPLE to die where they prefer is an important aim of palliative care ${ }^{1-3}$ and place of death is considered an indicator of quality of end-of-life care. ${ }^{4}$ There is strong evidence that many terminally ill people do not die where they would choose to. ${ }^{5}$ Most people prefer to die at home because of the psychological comfort, the presence of relatives and the feeling of control, ${ }^{6}$ although in cases of inaccurate pain control or a high burden of care on the family, many may prefer to die in an institution. ${ }^{6-9}$ In 2006, 57.7\% of the terminally ill in Belgium preferred to die at home, $30.9 \%$ in a care home, $4.7 \%$ in hospital, and $6.6 \%$ in a palliative care unit. ${ }^{8}$ Where someone dies depends on factors related to their medical condition, their sociodemographic characteristics, their personal preferences, their health care use, and their environment, including the social support and health care resources available. ${ }^{10-16}$

The association between involvement of palliative care services and place of death has not been investigated extensively before. The findings of the studies that do exist are equivocal, probably due to the heterogeneity of palliative care services, study populations, settings, and designs. ${ }^{17-25}$ Moreover, few studies have evaluated the relationship between involvement of palliative care services and place of death considering primary health care use, and none have evaluated the impact of a complete palliative care model, including all palliative care services for all patients in all health care settings, on place of death in a particular country.

\footnotetext{
${ }^{1}$ End-of-Life Care Research Group, Ghent University and Vrije Universiteit Brussel, Brussels, Belgium.

${ }^{2}$ Scientific Institute of Public Health, Department of Epidemiology, Brussels, Belgium.

${ }^{3}$ Department of Public and Occupational Health, EMGO Institute for Health and Care Research and Palliative Care Center of Expertise, VU University Medical Center, Amsterdam, The Netherlands.

Accepted July 27, 2010.
} 
Palliative care services in Belgium have been developing since 1985, and the 2002 law on palliative care provides for access to palliative care at the end of life. ${ }^{26}$ The objectives of palliative care policy are to integrate palliative care into general health care (the care usually provided by professional caregivers in different health care settings), primary care in particular, rather than to replace that with care delivered by palliative care specialists, and to support out-of-hospital death. ${ }^{2,3}$ Therefore, palliative care services have been set up to offer consultation and support for, and in principle only exceptionally to take over from, primary caregivers (i.e., general practioners [GPs] and home care nurses at home and GPs and care home nurses in care homes) in caring for the dying at home (known as multidisciplinary palliative home care teams) or in care homes (palliative care reference persons) and from medical and nursing staff in hospitals (multidisciplinary palliative support teams). In addition, small-scale inpatient palliative care units in or near hospitals were established that can be considered as a health care setting and thus as another possible location of death. ${ }^{2}$

The aim of this study is to examine, using nationwide retrospective data, the association between involvement of palliative care services in all health care settings and place of death, taking into account the personal characteristics of the deceased, environmental factors and general health care use. The research questions are: (1) what factors, related to personal characteristics, the environment, and general health care use, are related to place of death in Belgium and (2) is involvement of palliative care services associated with place of death?

\section{Methods}

We used retrospective data from a nationwide mortality follow-back study about palliative care services involvement and general health care use delivered in the last 3 months of life to people who died in 2005 or 2006. Data were collected within the SENTI-MELC study by the sentinel network of general practitioners, an epidemiologic surveillance network representative of all Belgian GPs, established in 1979 and covering $1.75 \%$ of the Belgian population. A sentinel network of GPs is a network of community-based physicians who monitor health problems on a continuing basis. ${ }^{27-29}$ All GPs were asked to report weekly, on a standardized registration form, every patient in their practice who had died during that week. The study population consists of people aged 1 year or older who were part of the GP practice and who had died nonsuddenly or expectedly as judged by the GP, i.e., those who can be considered potentially eligible for palliative care ${ }^{30}$ $(N=1690)$.

The dependent variable is place of death recoded into four categories: home, care home, hospital, and inpatient palliative care unit. The independent variables can be classified as personal, environmental, and health care use factors. ${ }^{10-13}$ The personal consist of the underlying cause of death, age, gender, educational attainment, and financial situation and the preference for place of death as communicated to the GP. The environmental factors include social support (living situation, involvement of informal care), health care resources (available hospital and care home beds), and the urbanization level of the place of residence. The health care use factors include the treatment goal and the use of general health care in the last 3 months of life, i.e., the level of GP and home care involvement, the number of hospital admissions (median length of stay of one hospital admission: 18 days; interquartile range: 9-31 days ${ }^{31}$ ), and the involvement of palliative care services, multidisciplinary palliative home care teams for those living at home, palliative care reference persons for those residing in care homes, and multidisciplinary palliative support teams for those living at home or in a care home admitted to hospital.

Bivariate associations between personal factors, environmental factors, the involvement of general health care and palliative care services and place of death were examined using cross tabulation and Pearson's $\chi^{2}$ tests.

Multivariate analyses using binomial logistic regression were performed separately for two subpopulations with respect to living situation, those living at home and those living in care homes, because they differ significantly regarding age, gender, and cause of death, and the palliative care services that are available to them are not the same. For both subpopulations, we examined the association of involvement of palliative care services available to them with place of death, taking in account personal, environmental and health care use factors.

For those living at home we examined the association of involvement of a multidisciplinary palliative home care team with home death, and the association of involvement of a multidisciplinary palliative home care team or a hospital palliative support team with death in an inpatient palliative care unit. For those residing at home and admitted to hospital at least once in the final 3 months, we examined the association with home death of the involvement of a palliative home care team or a hospital palliative support team.

For people residing in care homes, we examined the association of involvement of palliative care reference persons with death in their place of residence.

Because we were interested in the association of the level of GP involvement with place of death taking into account palliative care services involvement, we built up a regression model progressively to examine whether the expected association between high GP involvement and out-of-hospital death was adjusted when accounting for the involvement of palliative care services.

Significance was set at $p<0.05$. In multivariate analysis we used a stepwise forward likelihood ratio procedure to select model variables. Nagelkerke's $R^{2}$ was used to evaluate model fit and tolerance and the variance inflation factor (VIF) to examine multicollinearity. SPSS 17.0 (SPSS Inc., Chicago, IL) was used for statistical computations.

\section{Results}

Death at home occurred more often in those who died from cancer, were under 65 , male, had expressed a wish to die at home, lived in a multiperson household, received informal care often, and lived in a health care region with lower hospital bed availability (Table 1). Those who died from cardiovascular diseases, were under 85, male, did not express a preferred place of death, lived alone with little or no informal care and in regions with higher hospital bed availability more often died in hospital. Death in a care home occurred more often in those suffering from neurodegenerative diseases, being 85 or older, female, had lower educational attainment 


\begin{tabular}{|c|c|c|c|c|c|}
\hline \multirow[b]{3}{*}{ Personal and environmental factors } & \multirow[b]{3}{*}{$\mathrm{n}(\%)^{\mathrm{a}}$} & \multicolumn{4}{|c|}{ Place of death } \\
\hline & & Home & $\begin{array}{l}\text { Hospital (excl. } \\
\text { palliative care unit) }\end{array}$ & $\begin{array}{l}\text { Care } \\
\text { home }\end{array}$ & $\begin{array}{l}\text { Palliative } \\
\text { care unit }\end{array}$ \\
\hline & & & $\%$ & & \\
\hline $\begin{array}{l}\text { All deaths that occurred nonsuddenly or expectedly } \\
\text { Personal factors }\end{array}$ & 1690 & 23.8 & 39.3 & 26.7 & 10.1 \\
\hline $\begin{array}{l}\text { Underlying cause of death } \\
\text { Cardiovascular diseases } \\
\text { Malignancies } \\
\text { Respiratory diseases } \\
\text { Diseases of the nervous system } \\
\text { Stroke } \\
\text { Other }\end{array}$ & $\begin{aligned} 237 & (14.2) \\
725 & (43.4) \\
157 & (9.4) \\
71 & (4.2) \\
121 & (7.2) \\
360 & (21.5)\end{aligned}$ & $\begin{array}{r}17.7 \\
34.6 \\
18.5 \\
21.1 \\
9.1 \\
13.6\end{array}$ & $\begin{array}{l}50.6 \\
34.3 \\
44.6 \\
25.4 \\
40.5 \\
41.9\end{array}$ & $\begin{array}{l}29.1 \\
12.0 \\
35.0 \\
49.3 \\
43.0 \\
41.7\end{array}$ & $\begin{array}{r}2.5 \\
19.0 \\
1.9 \\
4.2 \\
7.4 \\
2.8\end{array}$ \\
\hline $\begin{array}{l}\text { Demographic variables } \\
\text { Age } \\
\text { 1-64 years } \\
\text { 65-84 years } \\
\text { 85+ years }\end{array}$ & $\begin{array}{l}199(12.0) \\
932(56.1) \\
530(31.9)\end{array}$ & $\begin{array}{l}34.7 \\
24.9 \\
17.0\end{array}$ & $\begin{array}{l}40.2 \\
43.9 \\
32.1\end{array}$ & $\begin{array}{r}5.5 \\
19.5 \\
47.0\end{array}$ & $\begin{array}{r}19.6 \\
11.7 \\
4.0\end{array}$ \\
\hline $\begin{array}{l}\text { Gender }^{\mathrm{b}} \\
\text { Male } \\
\text { Female }\end{array}$ & $\begin{array}{l}839(49.6) \\
851(50.4)\end{array}$ & $\begin{array}{l}28.0 \\
19.7\end{array}$ & $\begin{array}{l}44.3 \\
34.4\end{array}$ & $\begin{array}{l}17.4 \\
36.0\end{array}$ & $\begin{array}{r}10.3 \\
9.9\end{array}$ \\
\hline $\begin{array}{l}\text { Social Conditions } \\
\text { Educational Attainment } \\
\text { Elementary or lower } \\
\text { Lower secondary } \\
\text { Higher secondary } \\
\text { Higher }\end{array}$ & $\begin{array}{l}666(44.3) \\
425(28.2) \\
286(19.0) \\
128(8.5)\end{array}$ & $\begin{array}{l}22.2 \\
24.0 \\
26.9 \\
26.6\end{array}$ & $\begin{array}{l}38.6 \\
40.7 \\
40.6 \\
43.0\end{array}$ & $\begin{array}{l}31.5 \\
23.5 \\
19.6 \\
19.5\end{array}$ & $\begin{array}{r}7.7 \\
11.8 \\
12.9 \\
10.9\end{array}$ \\
\hline $\begin{array}{l}\text { Financial Situation }{ }^{\mathrm{b}} \\
\text { (very) Low } \\
\text { Average } \\
\text { (very) High }\end{array}$ & $\begin{array}{l}474(28.5) \\
859(51.6) \\
331(19.9)\end{array}$ & $\begin{array}{l}20.7 \\
25.4 \\
24.2\end{array}$ & $\begin{array}{l}38.4 \\
39.1 \\
42.6\end{array}$ & $\begin{array}{l}32.1 \\
24.8 \\
22.7\end{array}$ & $\begin{array}{r}8.9 \\
10.7 \\
10.6\end{array}$ \\
\hline $\begin{array}{l}\text { Patient's Expression of Preferences } \\
\text { Place of death preference expressed to } \mathrm{GP}^{\mathrm{b}} \\
\text { Home or with family member } \\
\text { Care home } \\
\text { Elsewhere (hospital, palliative care unit) } \\
\text { Not expressed }\end{array}$ & $\begin{aligned} & 416(26.1) \\
& 220(13.8) \\
& 77(4.8) \\
& 878(55.2)\end{aligned}$ & $\begin{array}{r}68.5 \\
0.5 \\
6.5 \\
7.2\end{array}$ & $\begin{array}{r}17.3 \\
6.4 \\
45.5 \\
60.5\end{array}$ & $\begin{array}{r}3.8 \\
92.7 \\
1.3 \\
22.9\end{array}$ & $\begin{array}{r}10.3 \\
0.5 \\
46.8 \\
9.5\end{array}$ \\
\hline $\begin{array}{l}\text { Environmental factors } \\
\text { Social Support }\end{array}$ & & & & & \\
\hline $\begin{array}{l}\text { Living situation } \\
\text { At home, alone } \\
\text { At home with others } \\
\text { Care home }\end{array}$ & $\begin{array}{l}315(18.7) \\
811(48.0) \\
562(33.3)\end{array}$ & $\begin{array}{r}25.1 \\
39.0 \\
1.4\end{array}$ & $\begin{array}{l}53.3 \\
46.1 \\
21.5\end{array}$ & $\begin{array}{r}4.1 \\
3.1 \\
73.7\end{array}$ & $\begin{array}{r}17.5 \\
11.8 \\
3.4\end{array}$ \\
\hline $\begin{array}{l}\text { Informal care involvement } \\
\text { None or very little } \\
\text { Sometimes } \\
\text { Often }\end{array}$ & $\begin{array}{r}171(10.7) \\
210(13.2) \\
1214(76.1)\end{array}$ & $\begin{array}{r}3.5 \\
9.0 \\
30.6\end{array}$ & $\begin{array}{l}48.5 \\
39.0 \\
36.5\end{array}$ & $\begin{array}{l}41.5 \\
44.3 \\
21.7\end{array}$ & $\begin{array}{r}6.4 \\
7.6 \\
11.2\end{array}$ \\
\hline $\begin{array}{l}\text { Availability of Health Care Resources } \\
\text { Availability of hospital beds } / 1000^{\mathrm{b}} \\
\quad<\text { median } \\
\quad \geq \text { median }\end{array}$ & $\begin{array}{l}952(56.5) \\
733(43.5)\end{array}$ & $\begin{array}{l}27.2 \\
19.6\end{array}$ & $\begin{array}{l}37.6 \\
41.6\end{array}$ & $\begin{array}{l}25.9 \\
27.6\end{array}$ & $\begin{array}{r}9.2 \\
11.2\end{array}$ \\
\hline $\begin{array}{l}\text { Availability of care home beds } / 1000 \geq 65 \text { years }^{\mathrm{b}} \\
\quad<\text { median } \\
\quad \geq \text { median }\end{array}$ & $\begin{array}{r}678(40.1) \\
1012(59.9)\end{array}$ & $\begin{array}{l}26.4 \\
22.1\end{array}$ & $\begin{array}{l}41.0 \\
38.2\end{array}$ & $\begin{array}{l}23.6 \\
28.9\end{array}$ & $\begin{array}{r}9.0 \\
10.8\end{array}$ \\
\hline $\begin{array}{l}\text { Urbanization level }^{\mathrm{b}} \\
\text { High } \\
\text { Average } \\
\text { Low }\end{array}$ & $\begin{array}{l}917(54.4) \\
436(25.9) \\
332(19.7)\end{array}$ & $\begin{array}{l}22.5 \\
24.5 \\
27.1\end{array}$ & $\begin{array}{l}40.2 \\
39.2 \\
37.0\end{array}$ & $\begin{array}{l}25.6 \\
27.8 \\
28.0\end{array}$ & $\begin{array}{r}11.7 \\
8.5 \\
7.8\end{array}$ \\
\hline
\end{tabular}

${ }^{\text {a } P r e s e n t e d ~ p e r c e n t a g e s ~ a r e ~ r o w ~ p e r c e n t a g e s, ~ e x c e p t ~ p e r c e n t a g e s ~ b e t w e e n ~ b r a c k e t s ~(c o l u m n ~ p e r c e n t a g e s) . ~ N u m b e r s ~ m i g h t ~ n o t ~ a d d ~ u p ~ t o ~} N$ because of missing values.

${ }^{b} p$ value $<0.001$ for $\chi^{2}$-statistic of association between cause of death, age, gender, place of death preference, living situation, informal care and place of death; $p$ value $<0.01$ for association between educational attainment, available hospital beds and place of death; $p$ value $<0.05$ for association between financial status, available care home beds, and place of death; $p$ value $>0.05$ for association between urbanization level and place of death.

GP, general practioner. 
Table 2. Health Care Use Factors Associated with Place of Death $(N=1690)$

\begin{tabular}{|c|c|c|c|c|c|}
\hline \multirow[b]{3}{*}{ Health care use factors } & \multirow[b]{3}{*}{$\mathrm{n}(\%)^{\mathrm{a}}$} & \multicolumn{4}{|c|}{ Place of death } \\
\hline & & Home & $\begin{array}{c}\text { Hospital (excl. } \\
\text { palliative care unit) }\end{array}$ & $\begin{array}{l}\text { Care } \\
\text { home }\end{array}$ & $\begin{array}{l}\text { Palliative } \\
\text { care unit }\end{array}$ \\
\hline & & & $\%$ & & \\
\hline \multicolumn{6}{|l|}{ Treatment goal in last 3 months ${ }^{\mathrm{b}}$} \\
\hline Cure/prolonging life & $280(17.0)$ & 11.4 & 73.2 & 14.3 & 1.1 \\
\hline Comfort/ palliation & $1365(83.0)$ & 26.8 & 30.9 & 30.0 & 12.2 \\
\hline \multicolumn{6}{|l|}{ GP involvement in last 3 months ${ }^{b}$} \\
\hline No involvement & $55(3.3)$ & 5.5 & 69.1 & 3.6 & 21.8 \\
\hline Sometimes & $352(21.3)$ & 15.9 & 55.7 & 15.1 & 13.4 \\
\hline Often & $1248(75.4)$ & 27.0 & 32.8 & 31.7 & 8.6 \\
\hline \multicolumn{6}{|c|}{ Home care involvement in last 3 months $s^{\mathrm{b}, \mathrm{c}}$} \\
\hline No involvement & $164(15.3)$ & 25.6 & 65.9 & 0.6 & 7.9 \\
\hline Sometimes & $164(15.3)$ & 32.3 & 51.8 & 2.4 & 13.4 \\
\hline Often & $747(69.5)$ & 39.2 & 42.3 & 4.3 & 14.2 \\
\hline \multicolumn{6}{|l|}{ Hospital admissions in last 3 months ${ }^{b}$} \\
\hline No hospital admissions & $686(40.6)$ & 39.9 & 0.9 & 48.7 & 10.5 \\
\hline One hospital admission & $824(48.8)$ & 13.2 & 64.0 & 12.9 & 10.0 \\
\hline Two or more hospital admissions & $180(10.7)$ & 11.1 & 73.3 & 6.7 & 8.9 \\
\hline
\end{tabular}

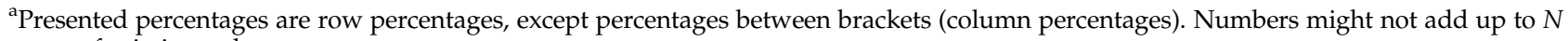
because of missing values.

${ }^{b} p$ value $<0.001$ for $\chi^{2}$-test of association between all health care use factors and place of death.

${ }^{\mathrm{c}}$ Applied for residents residing at home $(N=1126)$.

GP, general practitioner.

and lived in regions with higher care home bed availability. Death in a palliative care unit occurred more often in cases of cancer, in those under 65, having expressed a wish to die in hospital or a palliative care unit, lived alone, received informal care often and lived in a highly urbanized place.

Dying at home occurred more often in people whose treatment goal was comfort or palliation, whose GP was often involved in care during the last three months, who had professional home care often, and were not admitted to hospital in the final 3 months (Table 2). Dying in a hospital occurred more often in those whose treatment goal was cure or lifeprolonging and without GP or professional home care involvement. Death in a care home occurred more often if the treatment goal was comfort or palliation, if the GP was involved often and for those not admitted to hospital. Dying in a palliative care unit was associated with treatment aimed at comfort or palliation and with no or infrequent GP involvement.

Palliative care services were used by a minority (Table 3). Multidisciplinary palliative home care teams were involved in

Table 3. Involvement of Palliative Care Services Associated with Place of Death

\begin{tabular}{|c|c|c|c|c|c|}
\hline \multirow[b]{3}{*}{ Involvement of palliative care services in last 3 months } & \multirow[b]{3}{*}{$\mathrm{n}(\%)^{\mathrm{a}}$} & \multicolumn{4}{|c|}{ Place of death } \\
\hline & & Home & $\begin{array}{c}\text { Hospital (excl. } \\
\text { palliative care unit) }\end{array}$ & $\begin{array}{l}\text { Care } \\
\text { Home }\end{array}$ & $\begin{array}{l}\text { Palliative } \\
\text { care unit }\end{array}$ \\
\hline & & & $\%$ & & \\
\hline \multicolumn{6}{|c|}{ For patients residing at home $(N=1126)$} \\
\hline \multicolumn{6}{|c|}{ Involvement of a multidisciplinary palliative home care team ${ }^{\mathrm{b}}$} \\
\hline Involvement & $231(21.8)$ & 75.3 & 10.0 & 1.3 & 13.4 \\
\hline No involvement & $828(78.2)$ & 25.8 & 55.6 & 4.1 & 14.5 \\
\hline \multicolumn{6}{|c|}{ For patients residing in a care home $(N=562)$} \\
\hline \multicolumn{6}{|c|}{ Involvement of palliative care reference persons in care home $\mathrm{b}^{\mathrm{b}}$} \\
\hline Involvement & $157(29.1)$ & 0.6 & 2.5 & 96.2 & 0.6 \\
\hline No involvement & $382(70.9)$ & 1.8 & 27.2 & 66.2 & 4.7 \\
\hline \multicolumn{6}{|c|}{ For patients admitted to hospital at least once $(n=1004)$} \\
\hline \multicolumn{6}{|c|}{ Involvement of multidisciplinary palliative support team in hospital ${ }^{\mathrm{b}}$} \\
\hline Involvement & $115(12.4)$ & 10.4 & 62.6 & 6.1 & 20.9 \\
\hline No involvement & 813 (87.6) & 14.1 & 63.5 & 13.3 & 9.1 \\
\hline
\end{tabular}

${ }^{a}$ Presented percentages are row percentages, except percentages between brackets (column percentages). Numbers might not add up to $N$ because of missing values.

${ }^{\mathrm{b}} p$ value $<0.001$ for $\chi^{2}$-test of association between use of all palliative care services and place of death. 
Table 4. Adjusted Binomial Odds Ratios for Home Death vs. Hospital Death and Death in an Inpatient Palliative Care Unit vs. Hospital Death of Patients Residing at Home

\begin{tabular}{|c|c|c|c|}
\hline \multirow[b]{2}{*}{ Factors related to place of death } & $\begin{array}{l}\text { Home vs. hospital } \\
\text { death }(\mathrm{N}=750)^{\mathrm{a}}\end{array}$ & $\begin{array}{l}\text { Home vs. hospital death } \\
\text { for patients admitted to hospital } \\
\text { at least once in last } 3 \text { months }(N=533)^{\mathrm{b}, \mathrm{c}}\end{array}$ & $\begin{array}{l}\text { Death in a PCU vs. } \\
\text { hospital death } \\
\quad(\mathrm{N}=577)^{\mathrm{d}}\end{array}$ \\
\hline & \multicolumn{3}{|c|}{ OR $(95 \% C I)$} \\
\hline \multicolumn{4}{|l|}{ Personal factors } \\
\hline $\begin{array}{l}\text { Underlying cause of death } \\
\text { Other chronic life-limiting disease } \\
\text { Cancer }\end{array}$ & NS & NS & $\begin{array}{c}\text { Ref } \\
6.5(3.8-10.9)\end{array}$ \\
\hline $\begin{array}{l}\text { Age } \\
1-64 \text { years } \\
65-84 \text { years } \\
85+\text { years }\end{array}$ & NS & NS & NS \\
\hline $\begin{array}{l}\text { Gender } \\
\text { Male } \\
\text { Female }\end{array}$ & NS & NS & $\begin{array}{c}\text { Ref } \\
1.7(1.1-2.6)\end{array}$ \\
\hline $\begin{array}{l}\text { Financial situation } \\
\text { Low } \\
\text { Average } \\
\text { High }\end{array}$ & NS & NS & NS \\
\hline $\begin{array}{l}\text { Known preference for place of death? } \\
\text { Not known or other than home } \\
\text { Yes, at home }\end{array}$ & $\begin{array}{c}\text { Ref } \\
14.2(9.5-21.4)\end{array}$ & $\begin{array}{c}\text { Ref } \\
10.1(5.7-18.1)\end{array}$ & e \\
\hline \multicolumn{4}{|l|}{ Environmental factors } \\
\hline $\begin{array}{l}\text { Informal care involvement in last } 3 \text { months } \\
\text { No involvement or sometimes } \\
\text { Often }\end{array}$ & $\begin{array}{c}\text { Ref } \\
2.3(1.2-4.6)\end{array}$ & $\begin{array}{c}\text { Ref } \\
4.4(1.3-15.2)\end{array}$ & NS \\
\hline Hospital beds/1000 (continuous) & $0.8(0.6-0.9)$ & NS & NS \\
\hline Care home beds $/ 1000 \geq 65$ years (continuous) & NS & NS & NS \\
\hline $\begin{array}{l}\text { Urbanization level } \\
\text { Low or average } \\
\text { High }\end{array}$ & NS & NS & NS \\
\hline \multicolumn{4}{|l|}{ Health care use factors } \\
\hline $\begin{array}{l}\text { GP involvement in last } 3 \text { months } \\
\text { No involvement or sometimes } \\
\text { Often }\end{array}$ & NS & NS & NS \\
\hline $\begin{array}{l}\text { Home care involvement in last } 3 \text { months } \\
\text { No involvement or sometimes } \\
\text { Often }\end{array}$ & NS & NS & $\begin{array}{c}\text { Ref } \\
2.2(1.4-3.5)\end{array}$ \\
\hline \multicolumn{4}{|l|}{ Involvement of palliative care services } \\
\hline $\begin{array}{l}\text { Involvement of a multidisciplinary home care } t \\
\text { No involvement } \\
\text { Involvement }\end{array}$ & Ref & $\begin{array}{c}\text { Ref } \\
10.6(5.4-21.1)\end{array}$ & $\begin{array}{c}\text { Ref } \\
2.9(1.6-5.5)\end{array}$ \\
\hline $\begin{array}{l}\text { Involvement of a multidisciplinary palliative } \\
\text { support team in hospital } \\
\text { No involvement } \\
\text { Involvement }\end{array}$ & e & $\begin{array}{l}\text { Ref } \\
0.34(0.1-0.9)\end{array}$ & NS \\
\hline
\end{tabular}

${ }^{a}$ Nagelkerke's $R^{2}: 0.576$. No indications of severe multicollinearity were found: tolerance $>0.78, \mathrm{VIF}<1.28$.

${ }^{\mathrm{b}}$ Nagelkerke's $R^{2}$ : 0.494 . No indications of severe multicollinearity were found: tolerance $>0.83, \mathrm{VIF}<1.38$.

${ }^{\mathrm{c}}$ Median length of a hospital stay: 18 days, interquartile range: 9-31 days.

${ }^{\mathrm{d}}$ Nagelkerke's $R^{2}: 0.252$. No indications of severe multicollinearity were found: tolerance $>0.92, \mathrm{VIF}<1.21$.

${ }^{\mathrm{e}}$ Variable not involved in model.

PCU, palliative care unit; OR, odds ratio; CI, confidence interval; NS, not significant; GP, general practitioner; VIF, variance inflation factor.

$21.8 \%$, palliative care reference persons in care homes in $29.1 \%$ and multidisciplinary palliative support teams in hospitals in $12.4 \%$ of deaths. Home death occurred more frequently in people using a multidisciplinary home care team. Care home death happened more frequently if the palliative care reference persons were involved. For those admitted to hospital at least once in the final 3 months, death at home or in care homes occurred less frequently and death in an inpatient palliative care unit more frequently if a palliative support team of a hospital was involved.

Multivariate logistic regression, taking into account personal, environmental, and general health care use factors and palliative care services involvement, showed that involvement of palliative care services independently influenced place of death. For people living at home, home relative to hospital death was more likely if a multidisciplinary palliative 
home care team was involved (OR: 8.4, 95\% CI: 4.7-15.1), if they had expressed a preference for home death (OR: 14.2, 95\% CI: 9.5-21.4), used informal care often (OR: 2.3, 95\% CI:1.2-4.6), or lived in regions with lower hospital bed availability (OR: 0.8, 95\% CI: 0.6-0.9; Table 4). For those admitted to hospital at least once in the final 3 months, home death was less likely if a palliative support team in hospital was involved (OR: 0.3, 95\% CI: 0.1-0.9) and more likely if a multidisciplinary palliative home care team was involved (OR: 10.6, 95\% CI: 5.4-21.1), if a wish to die at home was expressed (OR: 10.1, 95\% CI: 5.7-18.1), or informal care was involved often (OR: 4.4, 95\% CI: 1.3-15.2). Death in an inpatient palliative care unit relative to a hospital was more likely

\section{Table 5. Adjusted Binomial Odds Ratios for Care Home Death vs. Hospital Death of Care Home Residents of 65 Years and Older}

\begin{tabular}{|c|c|}
\hline & $\begin{array}{l}\text { Care home } \\
\text { death vs. } \\
\text { hospital death } \\
(\mathrm{N}=443)^{\mathrm{a}}\end{array}$ \\
\hline Factors related to place of death & OR $(95 \% C I)$ \\
\hline \multicolumn{2}{|l|}{ Personal factors } \\
\hline $\begin{array}{l}\text { Underlying cause of death } \\
\text { Other chronic life-limiting disease } \\
\text { Cancer }\end{array}$ & $\begin{array}{c}\text { Ref } \\
2.5(1.1-5.9)\end{array}$ \\
\hline $\begin{array}{l}\text { Age } \\
\quad 65-84 \text { years } \\
85+\text { years }\end{array}$ & NS \\
\hline $\begin{array}{l}\text { Gender } \\
\text { Male } \\
\text { Female }\end{array}$ & $\begin{array}{c}\text { Ref } \\
1.8(1.0-3.0)\end{array}$ \\
\hline $\begin{array}{l}\text { Financial situation } \\
\text { Low } \\
\text { Average } \\
\text { High }\end{array}$ & NS \\
\hline $\begin{array}{l}\text { Known preference for place of death } \\
\text { Not known or other than care home } \\
\text { Yes, care home }\end{array}$ & $\begin{array}{c}\text { Ref } \\
10.4(4.4-24.9)\end{array}$ \\
\hline \multicolumn{2}{|l|}{ Environmental factors } \\
\hline $\begin{array}{l}\text { Patient had partner at time of death } \\
\text { No } \\
\text { Yes }\end{array}$ & NS \\
\hline Hospital beds/1000 (continuous) & NS \\
\hline $\begin{array}{l}\text { Care home beds } / 1000 \geq 65 \text { years } \\
\quad \text { (continuous) }\end{array}$ & NS \\
\hline $\begin{array}{l}\text { Urbanization level } \\
\text { Low } \\
\text { High }\end{array}$ & NS \\
\hline \multicolumn{2}{|l|}{ Health care use factors } \\
\hline $\begin{array}{l}\text { Level of GP involvement in last } 3 \text { months } \\
\text { No involvement or sometimes } \\
\text { Often }\end{array}$ & NS \\
\hline Involvement of palliative care services & \\
\hline $\begin{array}{l}\text { No Involvement } \\
\text { Involvement }\end{array}$ & $\begin{array}{c}\text { Ref } \\
9.4(3.3-26.7)\end{array}$ \\
\hline
\end{tabular}

${ }^{a}$ Nagelkerke's $R^{2}: 0.327$. No indications of severe multicollinearity were found: tolerance $>0.92$, VIF $<1.09$.

OR, odds ratio; CI, confidence interval; NS, not significant; GP, general practitioner; VIF, variance inflation factor. in cases involving a multidisciplinary palliative home care team (OR: 2.9, 95\% CI: 1.6-5.5), for patients with cancer (OR: 6.5, 95\% CI: 3.8-10.9), for women (OR: 1.7, 95\% CI: 1.1-2.6), or for those involving professional home care often (OR: 2.2, 95\% CI:1.4-3.5). We found no association between involvement of a palliative support team of a hospital and death in an inpatient palliative care unit.

People living in a care home were more likely to die there rather than in hospital if the palliative care reference persons were involved (OR: 9.4, 95\% CI: 3.3-26.7), if they preferred to die in the care home (OR: 10.4, 95\% CI: 4.4-24.9), if they had cancer (OR: 2.5, 95\% CI: 1.1-5.9), or if they were female (OR: 1.8, 95\% CI: 1.0-3.0; Table 5).

The unadjusted bivariate association found between frequent GP involvement and home death (OR: 3.4, 95\% CI: 2.54.8 ) or care home death (OR: 2.6, 95\% CI: 1.6-4.4), was adjusted completely when accounting for palliative care services involvement and the existence of a preference to die in the place of residence in case of care home residents, and significantly (from OR: 3.4, 95\% CI: $2.5-4.8$ to OR: $1.6,95 \%$ CI: $1.0-$ 2.6) in people living at home (not shown in table).

\section{Discussion}

Although involved with only a minority of terminally ill people, palliative care services prove to be a powerful predictor of place of death, along with the existence of a preference for place of death. The involvement of a multidisciplinary palliative home care team is strongly associated with higher chances of dying at home or in an inpatient palliative care unit. Care home residents referred to in-house palliative care reference persons are far more likely to die in their care home. In contrast, the intervention of a hospital multidisciplinary palliative support team does not support out-of-hospital death. Frequent involvement of the GP in the last 3 months of life was not directly associated with out-of-hospital death.

This study is to our knowledge the first to evaluate the whole palliative care services model on a nationwide scale with respect to one important outcome quality indicator. Using the sentinel network of GPs, the association of palliative care services involvement and place of death, taking general health care involvement and personal and environmental factors into account, is examined across all patient groups and care settings. This sentinel network is representative of all GPs in Belgium and has a long tradition in data collection with a stable group of participating GPs. Recall bias remains limited because of the requirement to register deaths weekly. ${ }^{27} \mathrm{Al}-$ though most GPs are usually kept informed about the care of their patients during hospital admissions, the involvement of palliative care services in hospitals and their impact on place of death might be underestimated. No information was available on the content of palliative care, the point in the disease course when it was begun and the period during which it was provided. The association of palliative care services involvement and place of death may be partly explained by confounders not included in our analyses, e.g., the preferences or coping skills of informal care givers or the patient's functional status, symptoms and other problems. Certain groups may have systematically had more chance of being referred to palliative care services, as a result of which place of death would not be a consequence of palliative care involvement but rather of particular patient characteristics. However, 
considering the amplitude of our findings, and the comprehensive set of factors controlled for, ${ }^{10}$ our results strongly suggest that palliative care services involvement affects place of death. Randomized prospective research designs would provide more decisive information as to the causal effect of palliative care services on place of death, but such designs would be difficult to implement in palliative care services research and as access to palliative care is a legal right in Belgium they could also be questioned legally and ethically.

Palliative care services at home and in care homes prove to be successful in supporting out-of-hospital death although they were used by only $21.8 \%$ and $29.1 \%$ of terminally ill people, possibly because of the insufficient capacity of these services, or the reluctance of the terminally ill, their families and professional caregivers to involve them. Where these services were involved, those living at home were 8.4 times more likely to die there and those in a care home 9.4 times more likely, regardless of the level of primary care involvement. The strong association of these palliative care services and place of death can probably be explained by several factors. Their 24 hours per day, 7 days per week availability ${ }^{18,25}$ and their expertise in treatment of pain and symptoms and in dealing with ethical issues can avoid hospitalization, and the comprehensive multidisciplinary support they provide, including emotional support to their clients and caregivers, may be a key element in facilitating home or care home death. ${ }^{32}$ The palliative care services we studied were implemented approximately 15 years ago and since then expertise in palliative care has developed rapidly. Treatment of pain and symptoms has become more technically sophisticated, requiring palliative care teams to be involved in treatment and not just in consulting and, since euthanasia became legal in Belgium in 2002,33 palliative care has also become more ethically complex, leading to an increase in all types of end-of-life decisions and requiring a complex decision-making process. ${ }^{34}$

The tendency for palliative care to face more complex challenges, the multidisciplinary nature of palliative care services and their permanent availability could explain the high impact they have on place of death, in contrast with high levels of GP involvement. Although in our final models we found that the level of GP involvement did not alter place of death, in contrast with previous findings, ${ }^{14,15}$ the gradual building-up of our models shows that highly involved GPs can play an intermediate role in supporting out-of-hospital death by exploring their patients' preferences and involving palliative care services in ways which will meet them. By doing so, however, their own influence on place of death is diminished.

In contrast to palliative care services operating where people live, we found that multidisciplinary palliative support teams in hospitals were not successful in supporting home death or death in an inpatient palliative care unit. It may be that people are systematically referred late to palliative support teams in hospitals due to an inadequate palliative care approach, resulting in lack of time to instigate a realistic plan for discharge from hospital. The success of multidisciplinary palliative home care teams in referring people to inpatient palliative units, in contrast with palliative support teams in hospitals, may be explained by the admission policies of palliative care units giving priority to those residing at home over those already in an institution, even a hospital, and because home care teams had been involved earlier in the disease trajectory with more time to plan future care for their clients.
Given the strong relationship we found between palliative care services involvement and place of death in Belgium, which may also apply to other countries with similar availability of palliative care services, health care policy-makers should consider strategies to encourage more intense cooperation between general health care practitioners and palliative care services providers. Integrating palliative care more systematically into the curricula of medical and nursing studies may be a good starting point. Future research should study the possible barriers of timely referral of hospitalized patients to palliative support teams in hospitals so that discharge from hospital to a more appropriate setting for end-oflife care remains feasible. The economic implications of dying in different care settings and the involvement of palliative care services both for patients and the health care system should be studied.

\section{Acknowledgments}

This study was supported by the Vrije Universiteit Brussel.

\section{Author Disclosure Statement}

No competing financial interests exist.

\section{References}

1. House of Commons Health Committee: Palliative Care. Fourth Report of Session 2003-04. www.publications .parliament.uk/pa/cm200304/cmselect/cmhealth/454/45402 .htm (Last accessed November 9, 2010).

2. Federal Evaluation Commission of Palliative Care: Evaluation Report of Palliative Care [in Dutch]. Brussels: Federal Public Service Health, Food Chain Safety and Environment, 2008. http://mailsystem.palliatief.be/accounts/15/ attachments/rapporten/fed_evaluatiecel_mai_2008_rapport_ 2008_nl.pdf (Last accessed November 9, 2010).

3. Clark D, ten Have H, Janssens R: Common threads? Palliative care service developments in seven European countries. Palliat Med 2000;14:479-490.

4. Teno JM, Clarridge BR, Casey V, Welch LC, Wetle T, Shield $\mathrm{R}$, Mor V: Family perspectives on end-of-life are at the last place of care. JAMA 2004;291:88-93.

5. Cohen J: End-of-Life Decisions and Place of Death in Belgium and Europe. Brussels: VUBPRESS, 2007.

6. Gott M, Seymour J, Bellamy G, Clark D, Ahmedzai S: Older people's views about home as a place of care at the end of life. Palliat Med 2004;18:460-467.

7. Wolff JL, Kasper JD, Shore AD: Long-term care preferences among older adults: A moving target? J Aging Soc Policy 2008;20:182-200.

8. Meeussen K, Van den Block L, Bossuyt N, Bilsen J, Echteld M, Van Casteren V, Deliens L: GPs' awareness of patients' preference for place of death. Br J Gen Pract 2009;59:665-670.

9. Abarshi E, Onwuteaka-Philipsen B, Donker G, Echteld M, Van den Block L, Deliens L: General practitioner awareness of preferred place of death and correlates of dying in a preferred place: A nationwide mortality follow-back study in the Netherlands. J Pain Symptom Manage 2009;38:568-577.

10. Gomes B, Higginson IJ: Factors influencing death at home in terminally ill patients with cancer: Systematic review. BMJ 2006;332:515-518A.

11. Grande GE, Addington-Hall JM, Todd CJ: Place of death and access to home care services: Are certain patient groups at a disadvantage? Soc Sci Med 1998;47:565-579. 
12. Gruneir A, Mor V, Weitzen S, Truchil R, Teno J, Roy J: Where people die: A multilevel approach to understanding influences on site of death in America. Med Care Res Rev 2007;64:351-378.

13. Tang ST, McCorkle R: Determinants of place of death for terminal cancer patients. Cancer Invest 2001;19:165-180.

14. Murray MA, Fiset V, Young S, Kryworuchko J: Where the dying live: A systematic review of determinants of place of end-of-life cancer care. Oncol Nurs Forum 2009;36:69-77.

15. Aabom B, Kragstrup J, Vondeling H, Bakketeig LS, Stovring $\mathrm{H}$ : Does persistent involvement by the GP improve palliative care at home for end-stage cancer patients? Palliat Med 2006;20:507-512.

16. Burge F, Lawson B, Johnston G, Cummings I: Primary care continuity and location of death for those with cancer. J Palliat Med 2003;6:911-918.

17. Ahlner-Elmqvist M, Jordhoy MS, Jannert M, Fayers P, Kaasa S: Place of death: Hospital-based advanced home care versus conventional care. A prospective study in palliative cancer care. Palliat Med 2004;18:585-593.

18. Kristjanson LJ, Cousins K, White K, Andrews L, Lewin G, Tinnelly C, Asphar D, Greene R: 1. Evaluation of a night respite community palliative care service. Int J Palliat Nurs 2004;10:84-90.

19. Hearn J, Higginson IJ: Do specialist palliative care teams improve outcomes for cancer patients? A systematic literature review. Palliat Med 1998;12:317-332.

20. McWhinney IR, Bass MJ, Orr V: Factors associated with location of death (home or hospital) of patients referred to a palliative care team. CMAJ 1995;152:361-367.

21. Fromme EK, Bascom PB, Smith MD, Tolle SW, Hanson L, Hickam DH, Osborne ML: Survival, mortality, and location of death for patients seen by a hospital-based palliative care team. J Palliat Med 2006;9:903-911.

22. Elsayem A, Smith ML, Parmley L, Palmer JL, Jenkins R, Reddy S, Bruera E: Impact of a palliative care service on inhospital mortality in a comprehensive cancer center. J Palliat Med 2006;9:894-902.

23. Jordhoy MS, Fayers $P$, Saltnes $T$, Ahlner-Elmqvist M, Jannert M, Kaasa S: A palliative-care intervention and death at home: a cluster randomised trial. Lancet 2000;356:888-893.

24. Currow DC, Burns CM, Abernethy AP: Place of death for people with noncancer and cancer illness in South Australia: A population-based survey. J Palliat Care 2008;24:144-150.

25. King G, Mackenzie J, Smith H, Clark D: Dying at home: Evaluation of a hospice rapid-response service. Int J Palliat Nurs 2000;6:280-287.
26. Wet betreffende palliatieve zorg 14 juni 2002 [Law Concerning Palliative Care June 14, 2002]. 2002022868, Belgisch Staatsblad 26 oktober 2002 [Belgian official collection of the laws October 26 2002], (2002)

27. Van den Block L, Van Casteren V, Deschepper R, Bossuyt N, Drieskens K, Bauwens S, Bilsen J, Deliens L: Nationwide monitoring of end-of-life care via the Sentinel Network of General Practitioners in Belgium: The research protocol of the SENTI-MELC study. BMC Palliat Care 2007;6:6.

28. Van den Block L, Deschepper R, Bilsen J, Van Casteren V, Deliens L: Transitions between care settings at the end of life in Belgium. JAMA 2007;298:1638-1639.

29. Van den Block L, Deschepper R, Bossuyt N, Drieskens K, Bauwens S, Van Casteren V, Deliens L: Care for patients in the last months of life: The Belgian Sentinel Network Monitoring End-of-Life Care study. Arch Intern Med 2008;168: 1747-1754.

30. Borgsteede SD, Deliens L, Francke AL, Stalman WA, Willems DL, van Eijk JT, van der Wal G: Defining the patient population: One of the problems for palliative care research. Palliat Med 2006;20:63-68.

31. Van den Block L, Bossuyt N, Van Casteren V, Deliens L: The Deathbed in Belgium. Results of the Senti-Melc Study 20052006. Brussels: Academic Scientific Publishers, 2007.

32. Higginson IJ, Costantini M: Dying with cancer, living well with advanced cancer. Eur J Cancer 2008;44:1414-1424.

33. Wet betreffende euthanasie 28 mei, 2002. Belgisch Staatsblad 22 juni 2002. [Law Concerning Euthanasia May 28, 2002]. 2002009590 Belgisch Staatsblad 22 juni 2002 [Belgian official collection of the Laws June 22, 2002], (2002).

34. Bilsen J, Cohen J, Chambaere K, Pousset G, OnwuteakaPhilipsen BD, Mortier F, Deliens L: Medical end-of-life practices under the euthanasia law in Belgium. N Engl J Med 2009;361:1119-1121.

Address correspondence to: Dirk Houttekier, M.A.

Vrije Universiteit Brussel

Faculty of Medicine and Pharmacy

End-of-Life Care Research Group

Ghent University $\mathcal{E}$ Vrije Universiteit Brussel

Laarbeeklaan 103

1090 Brussels

Belgium

E-mail: dirk.houttekier@vub.ac.be 